\title{
STREPTOCOCCUS PNEUMONIAE QUORUM SENSING AND BIOFILM FORMATION ARE AFFECTED BY THYMUS DAENENSIS, SATUREJA HORTENSIS, AND ORIGANUM VULGARE ESSENTIAL OILS
}

\author{
ARAM SHARIFi $^{1}$, Ali AHMADI ${ }^{2 *}$ and ABDOLMAJID MOHAMMADZADEH ${ }^{3}$ \\ ${ }^{1}$ Applied Microbiology Research Center, Systems Biology and Poisonings Center, \\ Baqiyatallah University of Medical Sciences, Tehran, Iran \\ ${ }^{2}$ Molecular Biology Research Center, Systems Biology and Poisonings Center, \\ Baqiyatallah University of Medical Sciences, Tehran, Iran \\ ${ }^{3}$ Department of Pathobiology, Faculty of Veterinary Science, Bu-Ali Sina University, \\ Hamedan, Iran
}

(Received: 24 October 2017; accepted: 15 January 2018)

The aim of this study was to investigate the effect of Thymus daenensis L., Satureja hortensis L., and Origanum vulgare L. essential oils (EOs) on the planktonic growth, biofilm formation, quorum sensing (QS), and competence system (CS) of Streptococcus pneumoniae. The anti-biofilm activity of EOs was determined by Microtiter-Plate Test (MtP) and scanning electron microscope (SEM). The QS and CS inhibitory activities were determined on the pre-grown biofilm by gene expression analysis using quantitative real-time RT-PCR. Using gas chromatography-mass spectrometry analysis, the major components of the tested EOs were detected. The MtP and SEM detected a significant inhibitory effect of the three EOs on biofilm formation at sub-minimum inhibitory concentrations (MICs). The most anti-biofilm activity was seen for T. daenensis. LuxS and $p f s$ genes (genes involved in QS) downregulated the following treatment with MIC/2 of Thymus and Satureja EOs. Thymol, carvacrol, p-cymene, pulegone, and 1,8-cineole were the major components of the tested EOs. The used EOs seem to be good candidates for preventing biofilm formation and subsequent colonization of $S$. pneumoniae. This study introduced $T$. daenensis and $S$. hortensis as new anti-biofilm and QS inhibitor agents with a natural origin.

Keywords: Streptococcus pneumoniae, biofilm formation, Thymus daenensis, Satureja hortensis, Origanum vulgare

*Corresponding author; E-mail: Aliahmadi@bmsu.ac.ir 


\section{Introduction}

Streptococcus pneumoniae (pneumococcus) is an important human pathogen, which causes community-acquired pneumonia, otitis media, meningitis, and sepsis especially in children [1]. Colonization and persistence of pneumococcus in the human nasopharynx are prerequisite factors for pneumococcal pathogenesis leading to a sessile microbial community referred to as "biofilm" [2, 3]. Biofilms are specialized bacterial structure communities that produce an extracellular matrix adhering to abiotic or biotic surfaces [4]. During the past two decades, many investigations were focused on bacterial biofilms, mainly due to the confirmed role of the biofilms in developing chronic and persistent infections [5]. According to Centers for Disease Control and Prevention, the biofilms are responsible for $65 \%$ of all infections [6]. Plant-derived natural compounds can inhibit the formation of microbial biofilms through various mechanisms, among which preventing from bacterial adhesion to living (or non-living) surfaces [7], and quorum sensing (QS) inhibitory (QSI) activity is most important $[8,9]$. The QS is the central system of biofilm formation that through interfering can abolish bacterial biofilms [10]. To evaluate the QSI activity, there are some phenotypic methods (such as violacein production by Chromobacterium violaceum CV026) and also genotypic methods (such as expression measuring of genes related to autoinducer-2 “AI-2") $[11,12]$. AI-2 is the most common and widespread QS signaling molecule in most bacteria including pneumococci. LuxS (S-ribosylhomocysteine lyase) and pfs (5'-methylthioadenosine) are the two important and necessary genes in AI-2 molecule synthesis pathway [11-14]. Some previous studies have investigated the QSI activity of pneumococci using luxS, $p f s$, and other AI-2-related genes expressions [14]. The second QS mechanism in the pneumococcus is ComABCD operon pathway. This operon produces a 17-residue signal peptide called competence stimulating peptide or CSP. In this pathway, comD gene encodes the CSP histidine kinase receptor and has an important role in Com QS pathway [15]. Previous studies have shown that some plant species, such as garlic, ginger, and turmeric, have QSI properties [16, 17]. The genus Thymus (commonly known as Thyme) contains hardy herbs, which can adapt to extreme climatic conditions. Leaves and flowering parts of this plants are traditionally used for various medical purposes, e.g., as an antispasmodic, antitussive, expectorant, carminative, anti-inflammatory, or tonic agent. Thymus daenensis L. is one of the Iranian endemic species, which have chemical composition and pharmacological activities similar to Thymus vulgaris L. [18].

Satureja hortensis L. (summer savory) that belongs to the Lamiaceae family is an important Iranian Satureja species, which is widely distributed in different parts of Iran. This plant is well known in Iranian traditional medicine and is used as carminative, astringent, tonic, and expectorant [19]. 
The genus Origanum (oregano) is one of over 200 genera in the Lamiaceae family. It is commonly used to flavor food or brew tea. The essential oil (EO) of oregano also has several alternate uses. The Origanum species have been used as spices and in folk medicine of many countries as diuretic, stomachic, antineuralgic, antitussive, expectorant, sedative, stimulant, carminative, antimicrobs, and antirheumatic [20].

Accordingly, this study was aimed to evaluate antibacterial, anti-biofilm, and anti-QS activity of $T$. daenensis, $S$. hortensis, and Origanum vulgare EOs against pneumococci. For assessment of anti-QS activity of these EOs, the expression level of $l u x S, p f s$, and $\operatorname{com} D$ was determined. These plants were chosen as they are endemic in many parts of Iran. These plants are extensively used in folk medicine and various systems of medicine and also some are used as topical antimicrobials.

\section{Materials and Methods}

EOs and gas chromatography-mass spectrometry (GC-MS) analysis

T. daenensis L. (herbarium code: MPH 2000), S. hortensis L. (herbarium code: MPH 2209), and O. vulgare L. (herbarium code: MPH 2215) were all obtained from Shahid Beheshti Plant Institute, Tehran, Iran. The samples were dried in shadow at room temperature for 10 days and the EOs were extracted from the dried leaves by the hydrodistillation method. Dried leaves of plants $(50 \mathrm{~g})$ were submitted to hydrodistillation, using Clevenger-type apparatus for $3 \mathrm{~h}$, according to the standard procedure [21]. The obtained EOs were dried over anhydrous sodium sulfate and stored in a sealed dark vials, then kept at $4{ }^{\circ} \mathrm{C}$ prior to further analysis. The yields of the EOs were $3.9 \%, 5.08 \%$, and $4.93 \%$ for T. daenensis, $S$. hortensis, and $O$. vulgare, respectively. These EOs were analyzed by GC-MS analysis using a Hewlett Packard 5972A mass selective detector coupled with a Hewlett Packard 6890 gas chromatograph equipped with a crosslinked 5\% PH ME siloxane HP-5MS capillary column $(30 \mathrm{~m} \times 0.25 \mathrm{~mm}$, film thickness $0.25 \mu \mathrm{m})$. The $\mathrm{GC}$ was carried out with below conditions: carrier gas, helium with a flow rate of $2 \mathrm{ml} / \mathrm{min}$; column temperature, $60-275^{\circ} \mathrm{C}$ at $4{ }^{\circ} \mathrm{C} / \mathrm{min}$; injector and detector temperatures, $280^{\circ} \mathrm{C}$; volume injected, $0.1 \mu \mathrm{l}$ of the oil; and split ratio, 1:25. The MS operating parameters were as follows: ionization potential, $70 \mathrm{eV}$; ion source temperature, $200{ }^{\circ} \mathrm{C}$; and resolution, 1,000. Identification of components in the oil was based on GC retention indices relative to $n$-alkanes and computer matching with the Wiley 275.L library, as well as by comparison of the fragmentation patterns of the mass spectra with those reported in the literature [22]. 
S. pneumoniae NCTC 7466 was obtained from Microbiology Department of Hamedan University of Medical Science, Hamedan, Iran. Ten pneumococcus clinical isolates, which had previously been isolated, were included in this study [23]. These pneumococcus isolates had been collected from patients admitted to the city hospitals and private laboratories in Tehran, Iran, over a period of 24 months, from 2011 to 2013. The bacteria were routinely grown in tryptic soy broth (TSB; BD Difco, Merck, Germany) or on blood agar plates supplemented with $5 \% \mathrm{v} / \mathrm{v}$ sheep blood at $37{ }^{\circ} \mathrm{C}$ in an atmosphere of $5 \% \mathrm{CO}_{2}$.

\section{Effect of EOs on planktonic cell growth}

The effect of EOs on planktonic bacteria was determined by broth microdilution method, as previously described [24]. Minimum inhibitory concentration (MIC) and minimum bactericidal concentration (MBC) of EOs were determined for planktonic $S$. pneumoniae. We prepared twofold serial dilutions of EOs $(0.156-20 \mu \mathrm{l} / \mathrm{ml})$ in TSB media, supplemented with $0.1 \%$ dimethyl sulfoxide (DMSO) to increase solubility of the EOs in the medium. Then, $100 \mu$ l of the solution was inoculated into each well of a 96-well flat-bottom microtiter plate (Nunc, South Korea). About $2 \mathrm{ml}$ of the bacterial inocula (0.5 McFarland standard) was added to each well. The controls were as: a negative control (medium, DMSO, and pneumococci); a blank medium with DMSO and EOs; and a positive control containing all medium, DMSO, pneumococci, and vancomycin. Plates were incubated aerobically at $37^{\circ} \mathrm{C}$ for $24 \mathrm{~h}$. The MIC was defined as the lowest concentration of EOs at which no visible growth was detected. For MBC determination, $5 \mu \mathrm{l}$ of inoculums (from the sample showing no visible growth) was incubated on blood agar medium and incubated at $37^{\circ} \mathrm{C}$ for $24 \mathrm{~h}$. The $\mathrm{MBC}$ was defined as the lowest concentration at which the original growth was reduced by $\geq 99.9 \%$.

\section{Effect of EOs on biofilm formation}

The effect of EOs on $S$. pneumoniae biofilm formation was determined by MtP assay [25]. The sterile 96-well polystyrene plates were filled with $98 \mu \mathrm{l}$ of TSB with $\mathrm{EO}$ in $\mathrm{MIC} / 2, \mathrm{MIC} / 4$, and $\mathrm{MIC} / 8$ concentrations and $0.1 \% \mathrm{DMSO}$ (five wells for each concentration). Then, $2 \mu \mathrm{l}$ (0.5 McFarland standard) of the inocula was added to each well. Negative and positive controls included bacteria with medium and vancomycin $(0.1 \mathrm{mg} / \mathrm{ml})$, respectively. The microplate was incubated without agitation for $24 \mathrm{~h}$ at $37^{\circ} \mathrm{C}$ and then the planktonic cells were removed. 
The surface-adhered cells were stained with $200 \mu \mathrm{l}$ of $0.1 \%$ safranin for $20 \mathrm{~min}$, and the excess dye was removed and the wells were washed. The safranin of the stained cells was solubilized with $100 \mu \mathrm{l}$ of $95 \%$ ethanol and the $\mathrm{OD}_{490 \mathrm{~nm}}$ was measured using a microplate reader. Each assay was repeated thrice and data are presented as the mean \pm SD. As a measure of efficacy, the percentage of inhibition was calculated using the mean absorbance of their controls by the following formula [26]:

Percentage of inhibition $=100-\left[\left(\mathrm{OD}_{490 \mathrm{~nm}}-\right.\right.$ treated wells $) /$

$\left(\right.$ mean $\mathrm{OD}_{490 \mathrm{~nm}}$ negative control well without antimicrobial agent $\left.) \times 100\right]$.

\section{Effect of EOs on formed biofilm}

The effect of EOs on pre-formed biofilm or biofilm disruption was determined by MtP assay according to Sieniawska et al.'s [25] method. The 24-h biofilms were allowed to establish in 96-well flat-bottom microtiter plate. In detail, each well was filled with $100 \mu \mathrm{l}$ TSB and $2 \mu \mathrm{l}$ of the bacteria as described for the MIC test. The microplates were incubated without agitation for $24 \mathrm{~h}$ at $37^{\circ} \mathrm{C}$ and then the broth culture was discarded and replaced with $100 \mu$ of TSB with $\mathrm{EO}$ in $\mathrm{MIC} / 2, \mathrm{MIC} / 4$, and MIC/8 concentrations and $0.1 \%$ DMSO. Five wells were considered for each concentration. Negative control wells were filled with $100 \mu \mathrm{l}$ of fresh broth medium and $0.1 \%$ DMSO. Then, microplates were subsequently incubated for a further $24 \mathrm{~h}$. Thereafter, the medium was gently aspirated and wells were rinsed thrice with phosphate-buffered saline and stained with $0.1 \%$ safranin. Then, $\mathrm{OD}_{490 \mathrm{~nm}}$ of the wells was calculated by a microplate reader. Each assay was repeated thrice and data were presented as the mean \pm SD. Biofilm disruption was determined using the following formula [26]:

Percentage of biofilm disruption $=100-\left[\left(\mathrm{OD}_{490 \mathrm{~nm}}-\right.\right.$ treated wells $) /$

$\left(\right.$ mean $\mathrm{OD}_{490 \mathrm{~nm}}$ negative control well without antimicrobial agent $\left.) \times 100\right]$.

\section{Scanning electron microscopic (SEM) analysis of pneumococcal biofilm}

Biofilms of $S$. pneumoniae were grown in 96-well microtiter plates, as described in Section "Effect of EOs on formed biofilm," with a piece of glass coverslip in each well. The control wells contained TSB, DMSO, and pneumococci, and the treated biofilm group contained the MIC/2 of each EOs in TSB medium containing $0.1 \%$ DMSO and pneumococci. After $24 \mathrm{~h}$ of incubation at $37{ }^{\circ} \mathrm{C}$, the samples were fixed at $2.5 \%$ buffered glutaraldehyde for $2.5 \mathrm{~h}$ 
followed by dehydration in graded ethanol. The samples were then dried at room temperature and glued onto stubs. Finally, the processed samples were sputter-coated with gold and examined in a JEOL JSM-840 SEM operating on an accelerating voltage of $15 \mathrm{kV}$.

\section{RNA extraction and cDNA synthesis}

To assess the effects of EOs on QS gene expressions of pneumococcal biofilms, we quantified the gene expressions of the biofilms grown with $\mathrm{MIC} / 2$ of these EOs versus the biofilms grown with DMSO and without EOs, using a quantitative real-time RT-PCR (qPCR) for $l u x S$, pfs, and comD QS and competence system (CS)-related genes. For RNA isolation, $S$. pneumoniae was grown with and without each EO in 96-well polystyrene tissue culture plates supplemented with TSB and incubated at $37{ }^{\circ} \mathrm{C}$ in an atmosphere of $5 \% \mathrm{CO}_{2}$ for $24 \mathrm{~h}$. After incubation, the plates were washed with deionized water to remove the planktonic cells and then the adherent cells were scraped and immediately processed for RNA extraction using CinnaPure RNA Extraction and Purification Kit (Cinnagen, Iran), according to the manufacturer's instructions. For removing DNA contamination, the extracted RNA was treated with RNase-free DNase I (Thermo Scientific, USA). The quality and quantity of the extracted RNA was determined by agarose gel electrophoresis and confirmed by measuring the absorbance at $260 \mathrm{~nm}$ using a Nanodrop spectrophotometer ND-1000 (Thermo Fisher Scientific, Wilmington, DE, USA). Extracted RNAs were stored at $-70{ }^{\circ} \mathrm{C}$ for next experiments. Then, the purified RNA was converted to cDNA, according to the manufacturer's instructions (cDNA Synthesis Kit, Takara, Japan), and stored at $-20{ }^{\circ} \mathrm{C}$ to use as the template for real time RT-PCR.

\section{Relative quantitative real-time $R T-P C R$}

SYBR Green real-time PCR Master Mix (Amplicon, Denmark) was used for real-time RT-PCR, according to the manufacturer's instructions. The primers (Takapouzist, Iran) used are listed in Table I. Real-time RT-PCR component and thermal conditions were carried out according to Yadav et al. [14]. The gyrB reference gene served as an internal control to normalize the expression levels of AI-2 synthesis-related genes [14]. The reaction was conducted in Corbett Life Science Rotor-Gene 6000 Cycler (Qiagen, Hilden, Germany). The relative gene expression was analyzed using the $2^{-\Delta \Delta C T}$ method [27]. 
Table I. Primers used in this study

\begin{tabular}{|c|c|c|c|}
\hline Genes & Primer $\left(5^{\prime}-3^{\prime}\right)$ & Product size $(b p)$ & References \\
\hline $\operatorname{lux} S$ & TATGTTCGCTTGATTGGG & 105 & [14] \\
\hline & GCCGGCAGTAGGGATAGAGT & & \\
\hline$p f_{s}$ & $\begin{array}{l}\text { TTGCTGCTATGCCAGAAGAA } \\
\text { TTCCCCAAAACAACTTGCTC }\end{array}$ & 76 & [14] \\
\hline $\operatorname{com} D$ & $\begin{array}{l}\text { GGATTTGGGACGGTTATTGTT } \\
\text { AATGTATAAGCACCAAAAACGAA }\end{array}$ & 110 & [14] \\
\hline $\operatorname{gyr} B$ & $\begin{array}{l}\text { CAGATCAAGAAATCAAACTCCAA } \\
\text { CAGCATCATCTACAGAAACTC }\end{array}$ & 172 & [14] \\
\hline
\end{tabular}

\section{Statistical analysis}

Data are expressed as mean $\pm \mathrm{SD}$. The statistical calculations were performed using graphpad prism software. An unpaired Student's $t$-test was used to analyze the data. A $p$ value of 0.05 was considered statistically significant. All the experiments were repeated thrice.

\section{Results}

GC-MS analysis

Our results showed that the EO of $T$. daenensis fresh leaves was mainly composed of thymol $(40.69 \%)$, followed by carvacrol $(30.28 \%)$, and p-cymene $(5.52 \%)$. The EO of $S$. hortensis fresh leaves contains thymol $(41.28 \%)$, $\gamma$-terpinene $(37.63 \%), p$-cymene $(12.2 \%)$, and $\alpha$-terpinene $(3.52 \%)$. For $O$. vulgare EO, the main compounds were pulegone (44.31\%), 1,8-cineole (17.47\%), and borneol (6.20\%).

\section{MIC and MBC determinations}

In vitro bacteriostatic and bactericidal properties of the three EOs were evaluated for the reference strain and clinical isolates by broth microdilution method. The results of MIC showed that all three EOs could prevent the growth of $S$. pneumoniae. The MICs of $T$. daenensis, $S$. hortensis, and $O$. vulgare EOs against $S$. pneumoniae planktonic cells were $0.625-1.25,2.5$, and $2.5-10 \mu 1 / \mathrm{ml}$, respectively. The MBC values were $1.25-2.5,2.5-5$, and $5-20 \mu \mathrm{l} / \mathrm{ml}$ for T. daenensis, S. hortensis, and O. vulgare EOs, respectively. 


\section{Effect of EOs on biofilm formation}

The results showed a dose-dependent manner in a decrease of biofilm formation in the presence of EOs (Figure 1). The T. daenensis $\mathrm{EO}$ was the most potent. At $\mathrm{MIC} / 2, \mathrm{MIC} / 4$, and $\mathrm{MIC} / 8$ concentrations, this $\mathrm{EO}$ was able to inhibit biofilm formation of $S$. pneumoniae. The inhibitory activity of $T$. daenensis $\mathrm{EO}$ in all three concentrations was significant $(p<0.001)$ comparing with the control (biofilms without EO). At MIC/8 and above concentration, the EOs of all three EOs significantly reduced the biofilm biomass. These results demonstrated that these EOs can significantly inhibit the biofilm formation for sub-MICs.

\section{Effect of EOs on formed biofilm}

As the EOs exhibited promising inhibitory potentials for biofilm formation, this experiment was further designed to assess their disruptive potential on preestablished biofilms. As a result, all three EOs had good potentials for disrupting S. pneumoniae-formed biofilm. As shown in detail in Figure 2, all EOs were able to significantly disrupt the biofilm at three sub-MICs (except MIC/8 for $S$. hortensis).

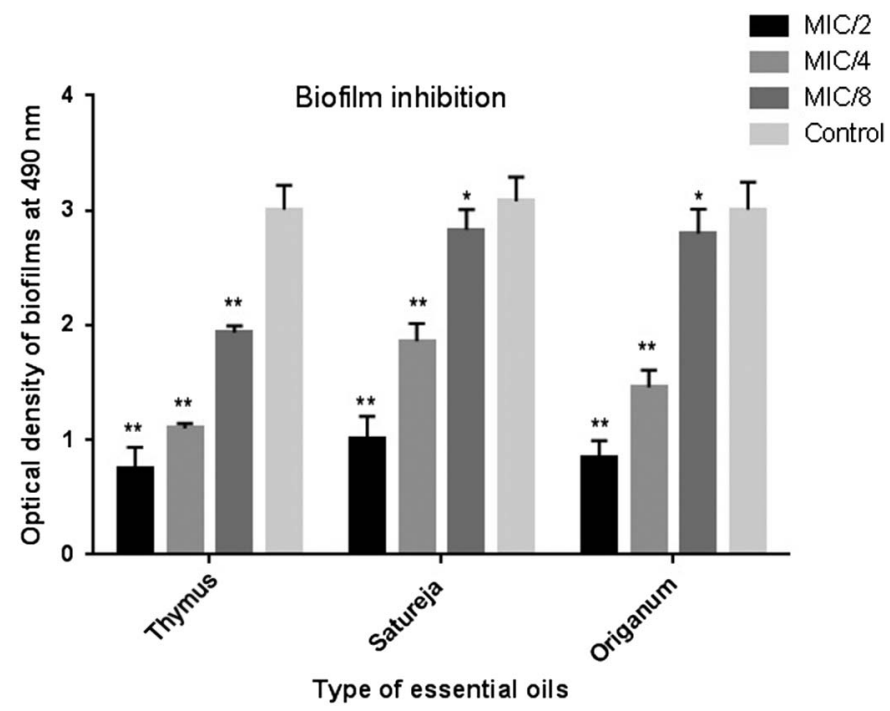

Figure 1. Inhibitory effect of essential oils at different concentrations on S. pneumonia (standard strain) biofilms. The error bars represent the standard deviation of three replicates $\left({ }^{*} p<0.05\right.$ and $* * p<0.001)$ 


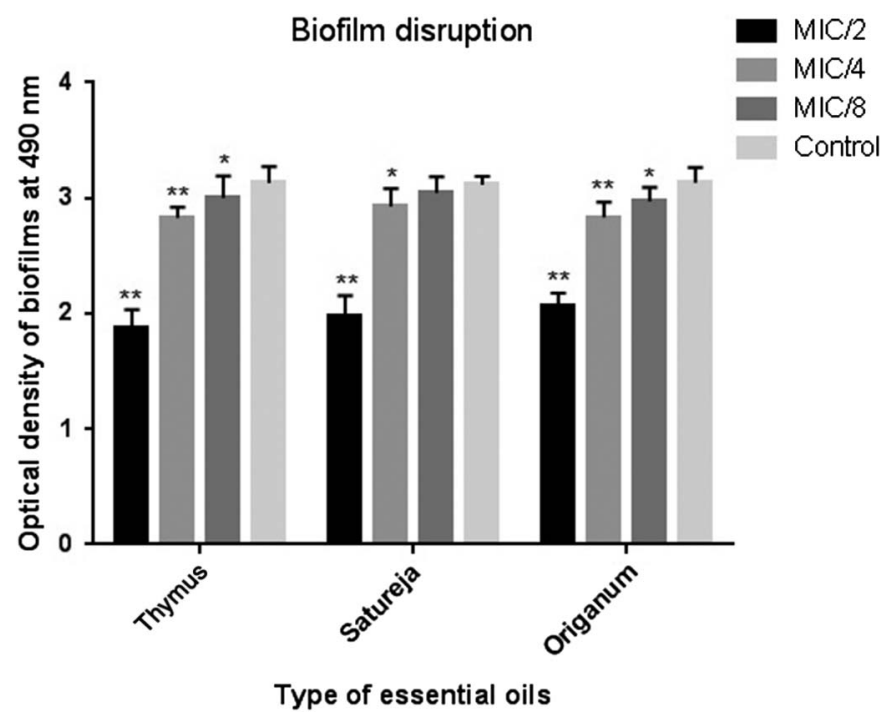

Figure 2. Disruption effect of essential oils at different concentrations on $S$. pneumoniae (standard strain) biofilm. The error bars represent the standard deviation of three replicates. $(* p<0.05$ and $* * p<0.001)$

\section{SEM analysis of pneumococcal biofilm}

The inhibition of pneumococcal biofilm formation by the EOs was observed by SEM, and the images showed a dense and uniform streptococcal biofilm that covered the coverslip-untreated biofilms (the control for biofilm formation) (Figure 3, Controls A and B). On other hand, the treated biofilms showed a significant reduction in the number of adherent bacteria and also the size of aggregates, which were reduced to small clusters or even single cells (Figure 3; Thymus, Satureja, and Origanum). These results confirmed the results of the crystal violet assay.

\section{Quantification of gene expression by quantitative real-time RT-PCR}

The effect of subinhibitory concentrations $(\mathrm{MIC} / 2)$ of $T$. daenensis, $S$. hortensis, and $O$. vulgare for $l u x S, p f s$, and comD gene expressions was measured on S. pneumoniae NCTC 7466 biofilm. We evaluated the transcript levels of luxS and $p f s$, because these two genes are critically involved in the AI-2 synthesis, and also tested $\operatorname{comD}$, which encodes a sensor histidine kinase and has a role in CS. In the biofilm phase, the gene expression values ranged from -4.55 - to 1.34 -fold 


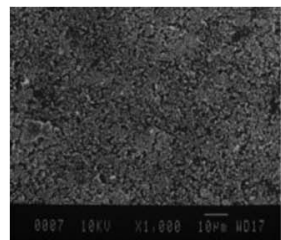

Control A

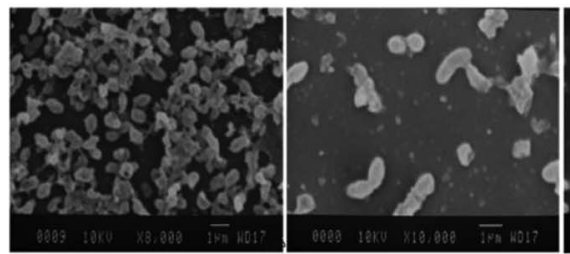

Control B

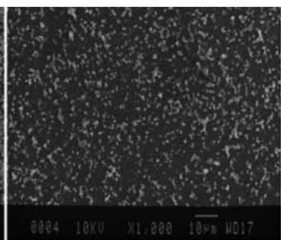

Thymus A

Thymus B

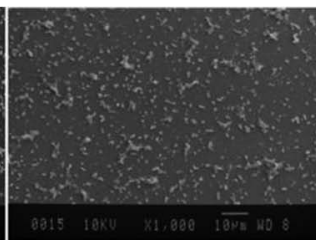

Satureja A

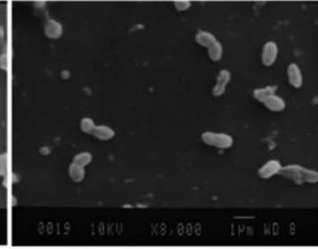

Satureja B

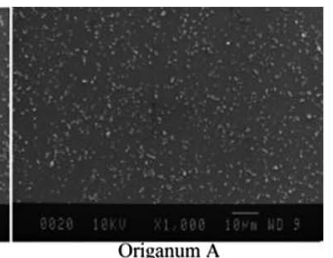

Origanum A

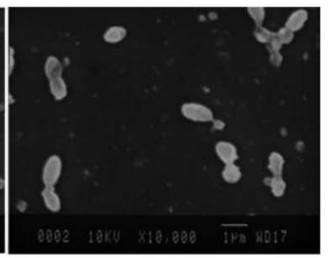

Origanum B

Figure 3. SEM images of biofilms upon the glass coverslips. The control image represents untreated

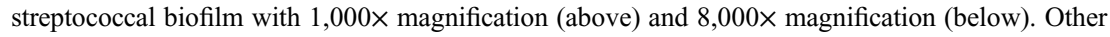
images showed treated streptococcal biofilm with studied EOs with various magnifications

changes after treatment with the EOs. The highest reduction in the AI-2-related gene expression was occurred in Satureja treatment. The gene expression of luxS and $p f s$ was significantly $(p<0.05)$ decreased ( 3.23 for $l u x S$ and 4.55 for $p f s)$ in the biofilm grown with Satureja. MIC/2 of Thymus EO significantly $(p<0.05)$ decreased the $l u x S$ and $p f s$ gene expressions. On the other hand, less than twofold changes in the gene expression of luxS, pfs, and comD were detected in treatment with MIC/2 of Origanum. More details related to fold changes in the gene expressions of pneumococcal biofilms grown with $\mathrm{MIC} / 2$ of these EOs were presented in Table II.

\section{Discussion}

In S. pneumoniae, the QS signal generated by AI-2 plays an important role in coordinating the spatial distribution of cells, aggregating the exopolysaccharides, and establishing three-dimensional structures of biofilms [28]. In this study, we have evaluated the effect of three plant EOs on planktonic and biofilm growth of S. pneumoniae. With respect to the important role of the QS system in biofilm formation, we investigated the effects of selected EOs on QS-related genes (luxS and $p f s$ ). Finally, the expression of CSP-1 receptor gene encoding ComD protein was tested in treatment with MIC/2 of selected EOs. This is the first report concerning the antimicrobial activity of $T$. daenensis, $S$. hortensis, and $O$. vulgare EOs against $S$. pneumoniae growing both as planktonic and biofilm phases. 
Table II. Gene expression analysis by real-time RT-PCR

\begin{tabular}{lccc}
\hline & \multicolumn{3}{c}{ Fold change in gene expression with $\mathrm{MIC} / 2$ of EOs } \\
\cline { 2 - 4 } Genes & Thymus & Satureja & Origanum \\
\hline \multirow{2}{*}{$\operatorname{luxS}$} & -2.27 & -3.23 & 1.29 \\
pfs & -2.56 & -4.55 & 1.65 \\
$\operatorname{comD}$ & 1.34 & -1.37 & -1.28 \\
\hline
\end{tabular}

Note: Fold changes in gene expression of biofilms grown with $\mathrm{MIC} / 2$ of EOs with respect to biofilms grown without EOs are shown in this table.

As shown in antibacterial tests, all three EOs exhibited a strong antibacterial activity against S.pneumoniae. The EO of T. daenensis was found to be the most effective with a minimum MIC $(0.625-1.25 \mu \mathrm{l} / \mathrm{ml})$. GC-MS analysis showed that the major components of $T$. daenensis were phenolic compounds, such as carvacrol and terpinene compounds, such as $\gamma$-terpinene and $\alpha$-terpinene. Previous studies reported that EOs containing phenolic and terpinene compounds have a strong antimicrobial activity [29]. EOs of $S$. hortensis and $O$. vulgare had good antibacterial activities against $S$. pneumoniae. EOs of these two plants had antibacterial compounds, including thymul and pulegone. Researchers believe that the main target of these components for bacterial killing or growth inhibition are cell wall, cytoplasmic membrane, and proteins embedded in the membrane [29]. However, these plant agents may also affect other bacterial components too $[29,30]$. For evaluating anti-biofilm activity, we used sublethal concentrations of EOs that are insufficient to neutralize the bacterium. As shown in Figures 1 and 2, all three EOs were able to significantly reduce biofilm formation as well as eradicate preestablished biofilms $(p<0.05)$. Our results indicated that the effects of the EOs against all the isolates were almost the same as those against the standard strain (NCTC 7466). Although there are many studies about other pathogenic bacteria, few studies have been done on the effects of EOs on pneumococcal biofilm formation. Karpanen et al. [31] investigated the activity of tea tree oil, eucalyptus oil, and thymol against both planktonic and biofilm forms of Staphylococcus epidermidis. They concluded that they significantly reduce biofilm formation in $S$. epidermidis and found that the most potent agent was thymol. Thymol is a phenolic compound that has amphipathic properties, which may alter the permeability of plasma membranes and disrupt bacterial adhesion to the surface, and subsequently reduce biofilm formation [32]. In addition, similar studies reported that terpinene compounds, such as $\alpha$-pinene can diminish biofilm formation by S. epidermidis [25], Bacillus cereus, Pichia anomala, and Pseudomonas putida [33]. In this study, GC-MS analysis revealed that the tested EOs contained thymol and terpinene compounds, which may be 
responsible for the most part of anti-biofilm and anti-adhesive properties. Previous studies demonstrated SEM as a valuable tool for qualitative and quantitative assessment of EOs' impacts on planktonic and biofilm forms of bacteria [14, 34]. Using SEM images, we demonstrated that for MIC/2, the concentration of all EOs could affect the bacterial biofilm and microcolony formation on the glass coverslip. As shown in Figure 3, the pneumococcal cells grown in EOs-free TSB medium were well-connected to each other and formed microcolonies, but these structures were rarely seen in EOs-treated pneumococcal cells. Absence of the normal biofilm structure in the treated group may be due to anti-adhesive properties of EO compounds, such as thymol [32], inhibition of exopolysaccharide production [35], or altering the biofilm-associated gene expression [36]. We used qPCR analysis for evaluation of QS-related gene expression for treatment by $\mathrm{MIC} / 2$ of the tested EOs. Previous studies used $\operatorname{luxS}$ and $p f s$ as functional genes in the QS pathway for both Gram-negative bacteria [37] and Gram-positive bacteria such as S. pneumoniae [14]. LuxS and Pfs are enzymes necessary for the production of AI-2 [12]. In this study, qPCR analysis revealed a significant downregulation of $l u x S$ and $p f s$ genes in pneumococcal biofilm grown under MIC/2 of Thymus and Satureja EOs (Table II). According to the role of the QS system in biofilm formation, toxin production, and antibiotic resistance, the QS is a main target for disrupting bacterial virulence [37]. On the other hand, researchers have demonstrated that mutation in or downregulation of $p f s$ and/or luxS genes can result in biofilm disruption as well as other adverse effects on pneumococcal physiology (such as cell growth, antibiotic resistance, and CS) [12, 14]. The present results showed that the gene expression of a competence-related gene $(c o m D)$ was changed on treatment by all three EOs, but the fold change of the expression was not significant. However, according to the impact of LuxS protein in CS [13], it seems that the tested EOs may indirectly affect this system through downregulating luxS gene. Finally, the results of qPCR demonstrated that a part of anti-pneumococcal biofilm properties of studied EOs, specially Thymus and Satureja, may be due to thier QSI activity. In addition, except for biofilm formation, the tested EOs may influence other QS-related virulence factors in pneumococci.

\section{Conclusions}

In conclusion, our results revealed that the investigated EOs seem to be suitable candidates for the prevention of biofilm formation and subsequent prevention of effective pneumococcal colonization. This study introduced T. daenensis and S. hortensis as new QSI agents with a natural origin. 
These findings suggest future studies for more examining the effect of these plants-based EOs on other pneumococcal virulence genes and also other pathogenic bacteria.

\section{Acknowledgements}

The authors would like to thank the Microbiology Department of Hamedan University of Medical Science, Hamedan, Iran, for providing the S. pneumoniae strain used in this study.

\section{Conflict of Interest}

No potential conflict of interest was reported by the authors.

\section{References}

1. Högberg, L., Geli, P., Ringberg, H., Melander, E., Lipsitch, M., Ekdahl, K.: Age- and serogroup-related differences in observed durations of nasopharyngeal carriage of penicillinresistant pneumococci. J Clin Microbiol 45, 948-952 (2007).

2. Kadioglu, A., Weiser, J. N., Paton, J. C., Andrew, P. W.: The role of Streptococcus pneumoniae virulence factors in host respiratory colonization and disease. Nat Rev Microbiol 6, 288-301 (2008).

3. Simell, B., Auranen, K., Käyhty, H., Goldblatt, D., Dagan, R., O'Brien, K. L.: The fundamental link between pneumococcal carriage and disease. Expert Rev Vaccines 11, 841-855 (2012).

4. Costerton, J. W., Stewart, P. S., Greenberg, E. P.: Bacterial biofilms: A common cause of persistent infections. Science 284, 1318-1322 (1999).

5. Donlan, R. M., Costerton, J. W.: Biofilms: Survival mechanisms of clinically relevant microorganisms. Clin Microbiol Rev 15, 167-193 (2012).

6. Packiavathy, I. A. S. V., Agilandeswari, P., Musthafa, K. S., Pandian, S. K., Ravi, A. V.: Antibiofilm and quorum sensing inhibitory potential of Cuminum cyminum and its secondary metabolite methyl eugenol against Gram negative bacterial pathogens. Food Res Int 45, 85-92 (2012).

7. Quave, C. L., Estévez-Carmona, M., Compadre, C. M., Hobby, G., Hendrickson, H., Beenken, K. E., Smeltzer, M. S.: Ellagic acid derivatives from Rubus ulmifolius inhibit Staphylococcus aureus biofilm formation and improve response to antibiotics. PLoS One 7, e28737 (2012).

8. Adonizio, A. L., Downum, K., Bennett, B. C., Mathee, K.: Anti-quorum sensing activity of medicinal plants in Southern Florida. J Ethnopharmacol 105, 427-435 (2006).

9. Bodini, S. F., Manfredini, S., Epp, M., Valentini, S., Santori, F.: Quorum sensing inhibition activity of garlic extract and p coumaric acid. Lett Appl Microbiol 49, 551-555 (2009). 
10. Brackman, G., Coenye, T.: Quorum sensing inhibitors as anti-biofilm agents. Curr Pharm Des 21, 5-11 (2015).

11. Chao, Y., Marks, L. R., Pettigrew, M. M., Hakansson, A. P.: Streptococcus pneumoniae biofilm formation and dispersion during colonization and disease. Front Cell Infect Microbiol 4, 194 (2015).

12. Vidal, J. E., Ludewick, H. P., Kunkel, R. M., Zähner, D., Klugman, K. P.: The LuxSdependent quorum-sensing system regulates early biofilm formation by Streptococcus pneumoniae strain D39. Infect Immun 79, 4050-4060 (2011).

13. Trappetti, C., Potter, A. J., Paton, A. W., Oggioni, M. R., Paton, J. C.: LuxS mediates irondependent biofilm formation, competence, and fratricide in Streptococcus pneumoniae. Infect Immun 79, 4550-4558 (2011).

14. Yadav, M. K., Chae, S. W., Song, J. J.: Effect of 5-azacytidine on in vitro biofilm formation of Streptococcus pneumoniae. Microb Pathog 53, 219-226 (2012).

15. Banerjee, G., Ray, A. K.: Quorum-sensing network-associated gene regulation in Grampositive bacteria. Acta Microbiol Immunol Hung 64, 439-453 (2017).

16. Koh, C. L., Sam, C. K., Yin, W. F., Tan, L. Y., Krishnan, T., Chong, Y. M., Chan, K. G.: Plant-derived natural products as sources of anti-quorum sensing compounds. Sensors 13, 6217-6228 (2013).

17. Vattem, D. A., Mihalik, K., Crixell, S. H., McLean, R. J. C.: Dietary phytochemicals as quorum sensing inhibitors. Fitoterapia 78, 302-310 (2007).

18. Zarshenas, M. M., Krenn, L.: A critical overview on Thymus daenensis Celak.: Phytochemical and pharmacological investigations. J Integr Med 13, 91-98 (2015).

19. Ghannadi, A.: Composition of the essential oil of Satureja hortensis L. seeds from Iran. J Essent Oil Res 14, 35-36 (2002).

20. Vazirian, M., Mohammadi, M., Farzaei, M. H., Amin, G., Amanzadeh, Y.: Chemical composition and antioxidant activity of Origanum vulgare subsp. vulgare essential oil from Iran. Res J Pharmacognosy 2, 41-46 (2015).

21. Council of Europe: European Pharmacopoeia, ( $6^{\text {th }}$ Edition). Council of Europe, Strasbourg, 2008.

22. Kabouche, A., Ghannadi, A., Kabouche, Z.: Thymus ciliates the highest thymol containing essential oil of the genus. Nat Prod Commun 4, 1251-1252 (2009).

23. Azadegan, A., Ahmadi, A., Lari, A.R., Talebi, M.: Detection of the efflux-mediated erythromycin resistance transposon in Streptococcus pneumoniae. Ann Lab Med 35, 57-61 (2015).

24. Olmaz, G., Ozen, F., Ekinci, Y., Bird, P. S., Korachi, M.: Inhibitory and disruptive effects of shiitake mushroom (Lentinula edodes) essential oil extract on oral biofilms. Jundishapur J Microbiol 6, e9058 (2013).

25. Sieniawska, E., Los, R., Baj, T., Malm, A., Glowniak, K.: Antimicrobial efficacy of Mutellina purpurea essential oil and $\alpha$-pinene against Staphylococcus epidermidis grown in planktonic and biofilm cultures. Ind Crops Prod 51, 152-157 (2013).

26. Onsare, J., Arora, D.: Antibiofilm potential of flavonoids extracted from Moringa oleifera seed coat against Staphylococcus aureus, Pseudomonas aeruginosa and Candida albicans. J Appl Microbiol 118, 313-325 (2015).

27. Livak, K. J., Schmittgen, T. D.: Analysis of relative gene expression data using real-time quantitative PCR and the $2^{-\Delta \Delta C T}$ method. Methods 25, 402-408 (2001). 
28. Allegrucci, M., Sauer, K.: Formation of Streptococcus pneumoniae non-phase-variable colony variants is due to increased mutation frequency present under biofilm growth conditions. J Bacteriol 190, 6330-6339 (2008).

29. Yadav, M. K., Go, Y. Y., Chae, S.-W., Song, J.-J.: The small molecule DAM inhibitor, pyrimidinedione, disrupts Streptococcus pneumoniae biofilm growth in vitro. PLoS One 10, e0139238 (2015).

30. Dorman, H., Deans, S.: Antimicrobial agents from plants: Antibacterial activity of plant volatile oils. J Appl Microbiol 88, 308-316 (2008).

31. Karpanen, T., Worthington, T., Hendry, E., Conway, B. R., Lambert, P. A.: Antimicrobial efficacy of chlorhexidine digluconate alone and in combination with eucalyptus oil, tea tree oil and thymol against planktonic and biofilm cultures of Staphylococcus epidermidis. J Antimicrob Chemother 62, 1031-1036 (2008).

32. Nostro, A., Roccaro, A. S., Bisignano, G., Marino, A., Cannatelli, M. A., Pizzimenti, F. C., Cioni, P. L., Procopio, F., Blanco, A. R.: Effects of oregano, carvacrol and thymol on Staphylococcus aureus and Staphylococcus epidermidis biofilms. J Med Microbiol 56, 519-523 (2007).

33. Kerekes, E. B., Deák, É., Takó, M., Tserennadmid, R., Petkovits, T., Vágvölgyi, C., Krisch, J.: Anti biofilm forming and anti quorum sensing activity of selected essential oils and their main components on food related micro organisms. J Appl Microbiol 115, 933-942 (2013).

34. da Silva Trentin, D., Giordani, R. B., Zimmer, K. R., Da Silva, A. G., da Silva, M. V., dos Santos Correia, M.: Potential of medicinal plants from the Brazilian semiarid region (Caatinga) against Staphylococcus epidermidis planktonic and biofilm lifestyles. J Ethnopharmacol 137, 327-335 (2011).

35. Swamy, M. K., Akhtar, M. S., Sinniah, U. R.: Antimicrobial properties of plant essential oils against human pathogens and their mode of action: An updated review. Evid Based Complement Alternat Med 2016, e3012462 (2016).

36. Kim, Y.-G., Lee, J.-H., Gwon, G., Kim, S.-I., Park, J.G., Lee, J.: Essential oils and eugenols inhibit biofilm formation and the virulence of Escherichia coli O157:H7. Sci Rep 6, 363377 (2016).

37. Lee, K.-M., Lim, J., Nam, S., Yoon, M. Y., Kwon, Y.-K., Jung, B. Y., Park, Y., Park, S., Yoon, S. S.: Inhibitory effects of broccoli extract on Escherichia coli O157:H7 quorum sensing and in vivo virulence. FEMS Microbiol Lett 321, 67-74 (2011). 